Exact Green function of Aharonov-Bohm billiard system

This content has been downloaded from IOPscience. Please scroll down to see the full text. 2001 J. Phys. A: Math. Gen. 342561

(http://iopscience.iop.org/0305-4470/34/12/304)

View the table of contents for this issue, or go to the journal homepage for more

Download details:

IP Address: 140.113.38.11

This content was downloaded on 28/04/2014 at 06:26

Please note that terms and conditions apply. 


\title{
Exact Green function of Aharonov-Bohm billiard system
}

\author{
Der-San Chuu and De-Hone Lin \\ Institute of Electro-Physics, National Chiao Tung University, Hsinchu 30043, Taiwan \\ E-mail: dhlin@cc.nthu.edu.tw
}

Received 12 April 2000, in final form 4 December 2000

\begin{abstract}
The exact Green functions of the relativistic spherical quantum AharonovBohm billiard systems in two- and three-dimensional spaces are given by the path integral approach. The transcendental equations for determining the energy spectra are discussed.
\end{abstract}

PACS numbers: 0365G, 0365B, 0365D

\section{Introduction}

Recently, the Aharonov-Bohm (AB) billiard systems have attracted extensive interest in the contexts of mesoscope, nonlinear and semiclassical dynamics. In this paper, we firstly calculate the Green functions of the relativistic two- and three-dimensional AB systems by the path integral approach. The exact Green functions of two- and three-dimensional relativistic spherical quantum AB billiard systems are then given for the first time by the closed formula of the perturbation technique. The transcendental equations for determining the energy spectra are given and the behaviour of spectra for large angular momentum are discussed.

\section{Green function of the two-dimensional AB billiard system}

The starting point is the path integral representation for the Green function of a relativistic particle in an external electromagnetic field [1,2]:

$G\left(\boldsymbol{x}_{b}, \boldsymbol{x}_{a} ; E\right)=\frac{\mathrm{i} \hbar}{2 m c} \int_{0}^{\infty} \mathrm{d} L \int \mathcal{D} \rho(\lambda) \Phi[\rho(\lambda)] \int \mathcal{D}^{D} x(\lambda) \exp \left\{-\mathcal{A}_{E}[\boldsymbol{x}, \dot{\boldsymbol{x}}] / \hbar\right\} \rho(0)$

with the action

$\mathcal{A}_{E}[\boldsymbol{x}, \dot{\boldsymbol{x}}]=\int_{\lambda_{a}}^{\lambda_{b}} \mathrm{~d} \lambda\left[\frac{m}{2 \rho(\lambda)} \dot{\boldsymbol{x}}^{2}(\lambda)-\mathrm{i}(e / c) \boldsymbol{A}(\boldsymbol{x}) \cdot \dot{\boldsymbol{x}}(\lambda)-\rho(\lambda) \frac{(E-V(\boldsymbol{x}))^{2}}{2 m c^{2}}+\rho(\lambda) \frac{m c^{2}}{2}\right]$ 
where $L$ is defined as

$$
L=\int_{\lambda_{a}}^{\lambda_{b}} \mathrm{~d} \lambda \rho(\lambda)
$$

in which $\rho(\lambda)$ is an arbitrary dimensionless fluctuating scale variable, $\rho(0)$ is the terminal point of the function $\rho(\lambda)$, and $\Phi[\rho(\lambda)]$ is some convenient gauge-fixing functional [1-3]. The only condition on $\Phi[\rho(\lambda)]$ is that

$$
\int \mathcal{D} \rho(\lambda) \Phi[\rho(\lambda)]=1
$$

$\hbar / m c$ is the well known Compton wavelength of a particle of mass $m, \boldsymbol{A}(\boldsymbol{x})$ and $V(\boldsymbol{x})$ stand for the vector and scalar potential of the system, respectively. $E$ is the system energy, and $x$ is the spatial part of the $(D+1)$ vector $x^{\mu}=(\boldsymbol{x}, \tau)$.

For the pure $\mathrm{AB}$ system under consideration, the scalar potential $V(\boldsymbol{x})=0$ and the vector potential reads

$$
\boldsymbol{A}(\boldsymbol{x})=2 g \frac{-y \hat{e}_{1}+x \hat{e}_{2}}{x^{2}+y^{2}}
$$

where $\hat{e}_{1,2}$ stand for the unit vectors along the $x, y$ axis, respectively. In the two-dimensional case, the functional $\Phi[\rho(\lambda)]$ can be taken as the $\delta$-functional $\delta[\rho-1]$ to fix the value of $\rho(\lambda)$ to unity [2]. The vector potential in equation (2.5) has another representation, obtained by introducing the azimuthal angle around the magnetic tube:

$$
\varphi(\boldsymbol{x})=\arctan (y / x) .
$$

The components of the vector potential turn into

$$
A_{i}=2 g \partial_{i} \varphi(\boldsymbol{x})
$$

and the magnetic interaction becomes

$$
\mathcal{A}_{\text {mag }}=-\hbar \beta_{0} \int_{0}^{L} \mathrm{~d} \lambda \dot{\varphi}(\lambda)
$$

where $\varphi(\lambda)=\varphi(\boldsymbol{x}(\lambda))$, and $\beta_{0}$ is the dimensionless number

$$
\beta_{0}=-\frac{2 e g}{\hbar c}
$$

The minus sign is a matter of convention. Since the particle orbits are present at all times, their worldlines in spacetime can be considered as being closed at infinity, and the integral

$$
n=\frac{1}{2 \pi} \int_{0}^{L} \mathrm{~d} \lambda \dot{\varphi}(\lambda)
$$

is the topological invariant with integer values of the winding number $n$. The AB magnetic interaction is purely topological, its value being

$$
\mathcal{A}_{\text {mag }}=-\hbar \beta_{0} 2 n \pi \text {. }
$$

The influence of $\mathrm{AB}$ effect on the entire system can be therefore considered by applying the Poisson summation formula

$$
\sum_{k=-\infty}^{\infty} f(k)=\int_{-\infty}^{\infty} \mathrm{d} y \sum_{n=-\infty}^{\infty} \mathrm{e}^{2 \pi n y \mathrm{i}} f(y)
$$

to the angular decomposition of equation (2.1). This leads to

$$
G\left(\boldsymbol{x}_{b}, \boldsymbol{x}_{a} ; E\right)=\frac{\mathrm{i} \hbar}{2 m c} \int_{0}^{\infty} \mathrm{d} L \mathrm{e}^{L \mathcal{E} / \hbar} \int_{-\infty}^{\infty} \mathrm{d} \alpha K\left(r_{b}, r_{a} ; L\right)_{\alpha} \cdot \sum_{n=-\infty}^{\infty} \frac{1}{2 \pi} \mathrm{e}^{\mathrm{i}\left(\alpha-\beta_{0}\right)\left(\varphi_{b}+2 n \pi-\varphi_{a}\right)}
$$


where the pseudoenergy $\mathcal{E}=\left(E^{2}-m^{2} c^{4}\right) / 2 m c^{2}$, and the radial pseudopropagator $K\left(r_{b}, r_{a} ; L\right)_{\alpha}$ has the representation

$$
K\left(r_{b}, r_{a} ; L\right)_{\alpha}=\frac{m}{\hbar} \frac{1}{L} \mathrm{e}^{-m\left(r_{b}^{2}+r_{a}^{2}\right) / 2 \hbar L} I_{\alpha}\left(\frac{m r_{b} r_{a}}{\hbar L}\right)
$$

with $I_{\alpha}$ the modified Bessel function. The sum over all $n$ in equation (2.13) forces $\alpha$ to be equal to $\beta_{0}$ which is modulo an arbitrary integral number. The Green function turns into

$$
G\left(\boldsymbol{x}_{b}, \boldsymbol{x}_{a} ; E\right)=\frac{\mathrm{i} \hbar}{2 m c} \int_{0}^{\infty} \mathrm{d} L \mathrm{e}^{L \mathcal{E} / \hbar} K\left(\boldsymbol{x}_{b}, \boldsymbol{x}_{a} ; L\right)
$$

in which $K\left(\boldsymbol{x}_{b}, \boldsymbol{x}_{a} ; L\right)$ is given by

$$
K\left(\boldsymbol{x}_{b}, \boldsymbol{x}_{a} ; L\right)=\sum_{n=-\infty}^{\infty} K\left(r_{b}, r_{a} ; L\right)_{n+\beta_{0}} \frac{1}{2 \pi} \mathrm{e}^{\mathrm{i} i\left(\varphi_{b}-\varphi_{a}\right)} .
$$

From equation (2.15), we observe that $K\left(\boldsymbol{x}_{b}, \boldsymbol{x}_{a} ; L\right)$ can be viewed as the propagator of the $\mathrm{AB}$ system with pseudoenergy $\mathcal{E}$. The entire Green function can be obtained by performing the integration.

Now let us begin to discuss the AB billiard system. With a method developed in $[4,5]$, the exact Green function of the quantum billiard for a spherically shaped, impenetrable wall located at the radius $r=a$ is given by the following formula:

$$
G^{(\text {wall })}\left(r_{b}, r_{a} ; E\right)=G\left(r_{b}, r_{a} ; E\right)-\frac{G\left(r_{b}, a ; E\right) G\left(a, r_{a} ; E\right)}{G(a, a ; E)}
$$

where $G\left(r_{b}, r_{a} ; E\right)$ is the Green function of unperturbed radial propagator. For the pure AB system under consideration, It can be obtained by noting equations (2.15) and (2.16) and reads

$$
G\left(r_{b}, r_{a} ; E\right)=\int_{0}^{\infty} \mathrm{d} L \mathrm{e}^{L \mathcal{E} / \hbar} \frac{m}{\hbar} \frac{1}{L} \mathrm{e}^{-m\left(r_{b}^{2}+r_{a}^{2}\right) / 2 \hbar L} I_{\left|n+\beta_{0}\right|}\left(\frac{m r_{b} r_{a}}{\hbar L}\right) .
$$

The integral can be performed by using the integral representation [6, p 200],

$\int_{0}^{\infty} \frac{\mathrm{d} z}{z} \mathrm{e}^{-p z-(a+b) / 2 z} I_{\nu}\left(\frac{a-b}{2 z}\right)=2 I_{v}(\sqrt{p}(\sqrt{a}-\sqrt{b})) K_{v}(\sqrt{p}(\sqrt{a}+\sqrt{b}))$

yielding

$$
\begin{aligned}
G\left(r_{b}, r_{a} ; E\right)= & \frac{2 m}{\hbar} I_{\left|n+\beta_{0}\right|}\left(\sqrt{-m \mathcal{E} / 2 \hbar^{2}}\left(r_{b}+r_{a}-\left|r_{b}-r_{a}\right|\right)\right) \\
& \times K_{\left|n+\beta_{0}\right|}\left(\sqrt{-m \mathcal{E} / 2 \hbar^{2}}\left(r_{b}+r_{a}+\left|r_{b}-r_{a}\right|\right)\right) .
\end{aligned}
$$

This gives the Green function with a wall located at $r=a$, for example, for $r_{a}<r_{b}<a$ :

$$
\begin{aligned}
G^{(\text {wall })}\left(r_{b}, r_{a} ; E\right) & =\frac{2 m}{\hbar}\left[I_{\left|n+\beta_{0}\right|}\left(\sqrt{-2 m \mathcal{E} / \hbar^{2}} a\right) K_{\left|n+\beta_{0}\right|}\left(\sqrt{-2 m \mathcal{E} / \hbar^{2}} r_{b}\right)-(K \leftrightarrow I)\right] \\
\times & \frac{I_{\left|n+\beta_{0}\right|}\left(\sqrt{-2 m \mathcal{E} / \hbar^{2}} r_{a}\right)}{I_{\left|n+\beta_{0}\right|}\left(\sqrt{-2 m \mathcal{E} / \hbar^{2}} a\right)} .
\end{aligned}
$$

The corresponding bound state energy spectra are given by the equation

$$
I_{\left|n+\beta_{0}\right|}\left(\sqrt{-2 m \mathcal{E} / \hbar^{2}} a\right)=0 .
$$

We see that the presence of the flux line in the circular billiard simply changes the order of the Bessel functions from the integer to fractional. If we assume that $-\beta_{0}$ can take a continuous 
range of values between 0 and 1 , the symmetry $\left|\beta_{0}\right| \leftrightarrow\left(1+\left|\beta_{0}\right|\right)$ in the quantum spectrum allows the restriction to $0 \leqslant\left|\beta_{0}\right| \leqslant 0.5$. For integer flux $\left|\beta_{0}\right|=0,1,2, \ldots$, the quantum spectrum is unaltered by the flux line. This is seen from the fact that for any integer value of $\beta_{0}$ the angular momentum gets to be redefined and the new set is isomorphic to the old one both in terms of the spectra and eigenstates. This mapping, however, has no classical analogue since the classically allowed angular momenta remain the same.

\section{Green function of the three-dimensional AB billiard system}

The path integral solution of the three-dimensional AB system can be solved by choosing the gauge-fixing condition in equation (2.4) as

$\int \mathcal{D} \rho(\lambda) \Phi[\rho(\lambda)]=\lim _{N \rightarrow \infty} \prod_{n=1}^{N+1}\left[\int \frac{\mathrm{d} w_{n}}{\left(2 \pi \hbar \epsilon_{n} r_{n} / m\right)^{1 / 2}}\right] \exp \left\{-\frac{1}{\hbar} \sum_{n=1}^{N+1} \frac{m}{2} \frac{\left(\Delta w_{n}\right)^{2}}{\epsilon_{n} r_{n}}\right\}$.

The unity condition is automatically satisfied. With this, the $\lambda$-sliced path integral of equation (2.1) turns into

$G\left(\boldsymbol{x}_{b}, \boldsymbol{x}_{a} ; E\right) \approx \frac{\hbar}{2 m c} \int_{0}^{\infty} \mathrm{d} L \int \mathrm{d} w_{b} \frac{r_{b}}{\left(\frac{2 \pi \hbar \epsilon_{b} r_{b}}{m}\right)^{2}} \prod_{n=1}^{N}\left[\int_{-\infty}^{\infty} \frac{\mathrm{d}^{4} x_{n}}{\left(\frac{2 \pi \hbar \epsilon_{n} r_{n}}{m}\right)^{2}}\right] \exp \left\{-\frac{1}{\hbar} \mathcal{A}_{E}^{N}\right\}$

where the sign $\approx$ in the above equation becomes an equality for $N \rightarrow \infty$, constant $\rho(0)$ is chosen as $r_{b}, \rho_{n}=r_{n}$ and the time-sliced action

$\mathcal{A}_{E}^{N}=\sum_{n=1}^{N+1}\left[\frac{m\left(\vec{x}_{n}-\vec{x}_{n-1}\right)^{2}}{2 \epsilon_{n} r_{n}}-\mathrm{i}(e / c) \boldsymbol{A}\left(\boldsymbol{x}_{n}\right) \cdot\left(\boldsymbol{x}_{n}-\boldsymbol{x}_{n-1}\right)-\epsilon_{n} r_{n} \frac{E^{2}}{2 m c^{2}}+\epsilon_{n} r_{n} \frac{m c^{2}}{2}\right]$

with the three-vectors $x$ of the kinematic term replaced with the four-vectors $\vec{x}$. To simplify the path integral, we invoke the KS transformation (see, for example, [8, p 500])

$$
\mathrm{d} \vec{x}=2 B(\vec{u}) \mathrm{d} \vec{u}
$$

where the $4 \times 4$ matrix $B(\vec{u})$ is chosen as

$$
B(\vec{u})=\left(\begin{array}{cccc}
u^{3} & u^{4} & u^{1} & u^{2} \\
u^{4} & -u^{3} & -u^{2} & u^{1} \\
u^{1} & u^{2} & -u^{3} & -u^{4} \\
u^{2} & -u^{1} & u^{4} & -u^{3}
\end{array}\right) .
$$

With this transformation, the volume element and velocity turn into

$$
\begin{aligned}
& \mathrm{d} \vec{x}=16 r^{2} \mathrm{~d} \vec{u} \\
& \left(\frac{\mathrm{d} \vec{x}}{\mathrm{~d} \lambda}\right)^{2}=4 \vec{u}^{2}\left(\frac{\mathrm{d} \vec{u}}{\mathrm{~d} \lambda}\right)^{2}=4 r\left(\frac{\mathrm{d} \vec{u}}{\mathrm{~d} \lambda}\right)^{2}
\end{aligned}
$$

and the $\mathrm{AB}$ magnetic interaction becomes

$$
\boldsymbol{A}(\boldsymbol{x}) \cdot \dot{\boldsymbol{x}}(\lambda)=-2 g \frac{y \dot{x}-x \dot{y}}{x^{2}+y^{2}}=-2 g\left[\frac{u^{1} \dot{u}^{2}-u^{2} \dot{u}^{1}}{\left(u^{1}\right)^{2}+\left(u^{2}\right)^{2}}+\frac{u^{4} \dot{u}^{3}-u^{3} \dot{u}^{4}}{\left(u^{3}\right)^{2}+\left(u^{4}\right)^{2}}\right] .
$$

We obtain a path integral equivalent to equation (3.2)

$$
G\left(x_{b}, x_{a} ; E\right)=\frac{\mathrm{i} \hbar}{2 m c} \int_{0}^{\infty} \mathrm{d} L G\left(\vec{u}_{b}, \vec{u}_{a} ; L\right)
$$

with the four-dimensional pseudopropagator

$$
G\left(\vec{u}_{b}, \vec{u}_{a} ; L\right)=\frac{1}{16} \int \frac{\mathrm{d} w_{b}}{r_{b}} \int \mathcal{D}^{4} u(\lambda) \exp \left\{\frac{\mathrm{i} \mathcal{A}_{E}\left[\vec{u}, \vec{u}^{\prime}\right]}{\hbar}\right\}
$$


where the action

$$
\mathcal{A}_{E}\left[\vec{u}, \vec{u}^{\prime}\right]=\int_{0}^{L} \mathrm{~d} \lambda\left[\frac{M \vec{u}^{\prime 2}}{2}-\mathrm{i}(e / c) \vec{A}(u) \cdot \vec{u}^{\prime}(\lambda)+\frac{M \omega^{2} \vec{u}^{2}}{2}\right]
$$

in which

$\vec{u}^{2}=\left(u^{1}\right)^{2}+\left(u^{2}\right)^{2}+\left(u^{3}\right)^{2}+\left(u^{4}\right)^{2}=r \quad M=4 m \quad \omega^{2}=\frac{E^{2}-m^{2} c^{4}}{4 m^{2} c^{2}}$

and $\vec{A}(u) \cdot \mathrm{d} \vec{u} / \mathrm{d} \lambda$ is given in (3.8). The functional measure in equation (3.10) has the $\lambda$-sliced representation

$$
\int \mathcal{D}^{4} u(\lambda) \approx \frac{1}{\left(\frac{2 \pi \hbar \epsilon_{b}}{M}\right)^{2}} \prod_{n=1}^{N}\left[\int_{-\infty}^{\infty} \frac{\mathrm{d}^{4} u_{n}}{\left(\frac{2 \pi \hbar \epsilon_{n}}{M}\right)^{2}}\right]
$$

Note that the path integral in equation (3.10) becomes separable like $R^{4} \rightarrow R^{2} \times R^{2}$ in which each $R^{2}$ has a dynamical model of a two-dimensional simple harmonic oscillator moving in the $\mathrm{AB}$ magnetic fields. Its Green function can be obtained by applying the procedures in equations (2.12) and (2.16) to the simple harmonic oscillator and is given by

$\frac{M \omega}{\hbar \sinh \omega L} \sum_{k=-\infty}^{\infty} \mathrm{e}^{\mathrm{i} k\left(\varphi_{b}-\varphi_{a}\right)} \exp \left\{-\frac{M \omega}{2 \hbar}\left(\sigma_{b}^{2}+\sigma_{a}^{2}\right) \operatorname{coth} \omega L\right\} I_{\left|k+\beta_{0}\right|}\left(\frac{M}{\hbar} \frac{\omega \sigma_{b} \sigma_{a}}{\sinh \omega L}\right)$

where $\sigma=\sqrt{x^{2}+y^{2}}$ is the radial length, $I_{\nu}$ is the modified Bessel function, and $\beta_{0} \equiv-2 e g / \hbar c$ as usual. Let us define the coordinate transformations between $\left\{u^{i} ; i=1, \ldots, 4\right\}$ and $\left\{\sigma_{i}, \varphi_{i} ; i=1,2\right\}$ as

$$
\begin{aligned}
& u^{1}=\sigma_{1} \sin \varphi_{1} \\
& u^{2}=\sigma_{1} \cos \varphi_{1} \\
& u^{3}=\sigma_{2} \cos \varphi_{2} \\
& u^{4}=\sigma_{2} \sin \varphi_{2} .
\end{aligned}
$$

We perform the path integral in equation (3.10) and obtain the entire Green function

$$
\begin{aligned}
G\left(\boldsymbol{x}_{b}, \boldsymbol{x}_{a} ; E\right)= & \frac{\mathrm{i} \hbar}{2 m c} \int_{0}^{\infty} \mathrm{d} L \frac{1}{16} \int \frac{\mathrm{d} w_{b}}{r_{b}}\left(\frac{M \omega}{\hbar \sinh \omega L}\right)^{2} \\
& \times \sum_{k_{1}=-\infty}^{\infty} \sum_{k_{2}=-\infty}^{\infty} \mathrm{e}^{\mathrm{i} k_{1}\left(\varphi_{1, b}-\varphi_{1, a}\right)} \mathrm{e}^{\mathrm{i} k_{2}\left(\varphi_{2, b}-\varphi_{2, a}\right)} \\
& \times \exp \left\{-\frac{M \omega}{2 \hbar}\left(\sigma_{1, b}^{2}+\sigma_{1, a}^{2}+\sigma_{2, b}^{2}+\sigma_{2, a}^{2}\right) \operatorname{coth} \omega L\right\} \\
& \times I_{\left|k_{1}+\beta_{0}\right|}\left(\frac{M}{\hbar} \frac{\omega \sigma_{1, b} \sigma_{1, a}}{\sinh \omega L}\right) I_{\left|k_{2}+\beta_{0}\right|}\left(\frac{M}{\hbar} \frac{\omega \sigma_{2, b} \sigma_{2, a}}{\sinh \omega L}\right) .
\end{aligned}
$$

To go further, we express the variables $\left(\sigma_{1}, \varphi_{1}, \sigma_{2}, \varphi_{2}\right)$ in terms of the Euler angle variables by defining

$$
\begin{array}{ll}
u^{1} & =\sqrt{r} \cos (\theta / 2) \cos [(\varphi+\gamma) / 2] \\
u^{2} & =-\sqrt{r} \cos (\theta / 2) \sin [(\varphi+\gamma) / 2] \\
u^{3} & =\sqrt{r} \sin (\theta / 2) \cos [(\varphi-\gamma) / 2] \\
u^{4} & =\sqrt{r} \sin (\theta / 2) \sin [(\varphi-\gamma) / 2]
\end{array} \quad\left(\begin{array}{c}
0 \leqslant \theta \leqslant \pi \\
0 \leqslant \varphi \leqslant 2 \pi \\
0 \leqslant \gamma \leqslant 4 \pi
\end{array}\right)
$$


and identify

$$
\begin{aligned}
& \sigma_{1}=\sqrt{r} \cos (\theta / 2) \\
& \varphi_{1}=(\varphi+\gamma+\pi) / 2 \\
& \sigma_{2}=\sqrt{r} \sin (\theta / 2) \\
& \varphi_{2}=(\varphi-\gamma) / 2 .
\end{aligned}
$$

The integral variable $\int \mathrm{d} w_{b} / r_{b}$ in equation (3.16) turns into

$$
\begin{aligned}
\mathrm{d} w & =2\left(u^{2} \mathrm{~d} u^{1}-u^{1} \mathrm{~d} u^{2}+u^{4} \mathrm{~d} u^{3}-u^{3} \mathrm{~d} u^{4}\right) \\
& =r(\cos \theta \mathrm{d} \varphi+\mathrm{d} \gamma)
\end{aligned}
$$

and due to the $\boldsymbol{x}$ the angles $(\theta, \varphi)$ remain fixed during the $w$ integration. Then one can change the $w_{b}$-integration into the $\gamma_{b}$-integration whose result is easily represented as the Kronecker delta $\delta_{k_{1}, k_{2}}$. Hence, we carry out $k_{2}$-summation and finally derive

$$
\begin{aligned}
G\left(\boldsymbol{x}_{b}, \boldsymbol{x}_{a} ; E\right)= & \frac{\mathrm{i} \hbar}{2 m c} \frac{m^{2} \omega}{\pi \hbar^{2}} \sum_{k=-\infty}^{\infty} \mathrm{e}^{\mathrm{i} k\left(\varphi_{b}-\varphi_{a}\right)} \\
& \times \int_{0}^{\infty} \mathrm{d} \eta \frac{1}{\sinh ^{2} \eta} \mathrm{e}^{-\frac{M \omega}{2 \hbar}\left(r_{b}+r_{a}\right) \operatorname{coth} \eta} I_{\left|k+\beta_{0}\right|}\left(\frac{M \omega \sqrt{r_{b} r_{a}}}{\hbar \sinh \eta} \cos \theta_{b} / 2 \cos \theta_{a} / 2\right) \\
& \times I_{\left|k+\beta_{0}\right|}\left(\frac{M \omega \sqrt{r_{b} r_{a}}}{\hbar \sinh \eta} \sin \theta_{b} / 2 \sin \theta_{a} / 2\right)
\end{aligned}
$$

where we have defined the new variable $\eta=\omega L$. The product of modified Bessel functions can be simplified by making use of the addition theorem [9]

$$
\begin{aligned}
& I_{v}(z \sin \alpha / 2 \sin \beta / 2) I_{\mu}(z \cos \alpha / 2 \cos \beta / 2) \\
& =\frac{2}{z}(\sin \alpha / 2 \sin \beta / 2)^{v}(\cos \alpha / 2 \cos \beta / 2)^{\mu} \sum_{l=0}^{\infty} \frac{l ! \Gamma(l+\mu+v+1)(2 l+\mu+v+1)}{\Gamma(l+\mu+1) \Gamma(l+v+1)} \\
& \quad \times I_{2 l+\mu+v+1}(z) P_{l}^{(\mu, v)}\left(\cos \theta_{b}\right) P_{l}^{(\mu, v)}\left(\cos \theta_{a}\right)
\end{aligned}
$$

where $P_{l}^{(\mu, v)}$ is the Jacobi polynomial (see, for example, [9, $\left.\mathrm{p} 209\right]$ ). The Green function in equation (3.20) becomes

$$
\begin{aligned}
G\left(\boldsymbol{x}_{b}, \boldsymbol{x}_{a} ; E\right)= & \frac{\mathrm{i} \hbar}{2 m c} \frac{m}{2 \pi \hbar \sqrt{r_{b} r_{a}}} \sum_{k=-\infty}^{\infty} \sum_{l=0}^{\infty} \mathrm{e}^{\mathrm{i} k\left(\varphi_{b}-\varphi_{a}\right)} \\
& \times\left(\cos \theta_{b} / 2 \cos \theta_{a} / 2 \sin \theta_{b} / 2 \sin \theta_{a} / 2\right)^{\left|k+\beta_{0}\right|} \\
& \times \frac{l ! \Gamma\left(l+2\left|k+\beta_{0}\right|+1\right)\left(2 l+2\left|k+\beta_{0}\right|+1\right)}{\Gamma^{2}\left(l+\left|k+\beta_{0}\right|+1\right)} \\
& \times\left\{\int_{0}^{\infty} \mathrm{d} \eta \frac{1}{\sinh \eta} \mathrm{e}^{-\frac{M \omega}{2 \hbar}\left(r_{b}+r_{a}\right) \operatorname{coth} \eta} I_{2 l+2\left|k+\beta_{0}\right|+1}\left(\frac{M \omega \sqrt{r_{b} r_{a}}}{\hbar \sinh \eta}\right)\right\} \\
& \times P_{l}^{\left(\left|k+\beta_{0}\right|,\left|k+\beta_{0}\right|\right)}\left(\cos \theta_{b}\right) P_{l}^{\left(\left|k+\beta_{0}\right|,\left|k+\beta_{0}\right|\right)}\left(\cos \theta_{a}\right) .
\end{aligned}
$$

This integral can be performed by noting the equality [3]

$$
\int_{0}^{\infty} \mathrm{d} z \frac{1}{\sinh z} \mathrm{e}^{-\frac{M \omega}{2 \hbar}\left(r_{b}+r_{a}\right) \operatorname{coth} z} I_{\nu}\left(\frac{\frac{M \omega}{\hbar} \sqrt{r_{b} r_{a}}}{\sinh z}\right)=\frac{1}{2} \int_{0}^{\infty} \frac{\mathrm{d} S}{S} \mathrm{e}^{-\frac{\varepsilon}{\hbar} S} \mathrm{e}^{-m\left(r_{b}^{2}+r_{a}^{2}\right) / 2 \hbar S} I_{\nu / 2}\left(\frac{m}{\hbar} \frac{r_{b} r_{a}}{S}\right)
$$


where $\mathcal{E}$ is defined as $\left(m^{2} c^{4}-E^{2}\right) / 2 m c^{2}$. We finally obtain the exact Green function of the relativistic three-dimensional $\mathrm{AB}$ effect:

$$
\begin{aligned}
G\left(\boldsymbol{x}_{b}, \boldsymbol{x}_{a} ; E\right)= & \frac{\mathrm{i} \hbar}{2 m c} \sum_{k=-\infty}^{\infty} \sum_{l=0}^{\infty} \mathrm{e}^{\mathrm{i} k\left(\varphi_{b}-\varphi_{a}\right)}\left(\cos \theta_{b} / 2 \cos \theta_{a} / 2 \sin \theta_{b} / 2 \sin \theta_{a} / 2\right)^{\left|k+\beta_{0}\right|} \\
& \times \frac{m}{2 \pi \hbar \sqrt{r_{b} r_{a}}} \frac{l ! \Gamma\left(l+2\left|k+\beta_{0}\right|+1\right)\left(2 l+2\left|k+\beta_{0}\right|+1\right)}{\Gamma^{2}\left(l+\left|k+\beta_{0}\right|+1\right)} \\
& \times\left\{I_{v}\left[\sqrt{\frac{m \mathcal{E}}{2 \hbar^{2}}}\left(\left(r_{b}+r_{a}\right)-\left|r_{b}-r_{a}\right|\right)\right] K_{v}\left[\sqrt{\frac{m \mathcal{E}}{2 \hbar^{2}}}\left(\left(r_{b}+r_{a}\right)+\left|r_{b}-r_{a}\right|\right)\right]\right\} \\
& \times P_{l}^{\left(\left|k+\beta_{0}\right|,\left|k+\beta_{0}\right|\right)}\left(\cos \theta_{b}\right) P_{l}^{\left(\left|k+\beta_{0}\right|,\left|k+\beta_{0}\right|\right)}\left(\cos \theta_{a}\right)
\end{aligned}
$$

with $v=l+\left|k+\beta_{0}\right|+1 / 2$. It is worth noting that there exist no bound states in the pure AB effect. This is reasonable, since we are treating a scattering system.

According to the orthogonality relations of Jacobi polynomials [9, $\mathrm{p} 212]$

$$
\begin{aligned}
\int_{-1}^{-1} \mathrm{~d} x(1-x)^{\alpha}(1+x)^{\beta} P_{n}^{(\alpha, \beta)}(x) P_{m}^{(\alpha, \beta)}(x) \\
=\frac{2^{\alpha+\beta+1}}{\alpha+\beta+2 n+1} \frac{\Gamma(\alpha+n+1) \Gamma(\beta+n+1)}{n ! \Gamma(\alpha+\beta+n+1)} \delta_{m, n}
\end{aligned}
$$

we find the radial Green function of the relativistic three-dimensional AB effect

$$
\begin{aligned}
G\left(r_{b}, r_{a} ; E\right)= & \frac{2 m}{\hbar \sqrt{r_{b} r_{a}}}\left\{I_{\nu}\left[\sqrt{\frac{m \mathcal{E}}{2 \hbar^{2}}}\left(\left(r_{b}+r_{a}\right)-\left|r_{b}-r_{a}\right|\right)\right]\right. \\
& \left.\times K_{v}\left[\sqrt{\frac{m \mathcal{E}}{2 \hbar^{2}}}\left(\left(r_{b}+r_{a}\right)+\left|r_{b}-r_{a}\right|\right)\right]\right\} .
\end{aligned}
$$

By applying the method developed in $[4,5]$ again, the effect of a spherically shaped impenetrable wall located at the radius $r=a$ can be studied via equation (2.17). This gives, for example, for $r_{a}<r_{b}<a$, the exact Green function

$$
\begin{aligned}
G^{(\text {wall })}\left(r_{b}, r_{a} ; E\right) & =\frac{2 m}{\hbar \sqrt{r_{b} r_{a}}}\left[I_{\nu}\left(\sqrt{2 m \mathcal{E} / \hbar^{2}} a\right) K_{\nu}\left(\sqrt{2 m \mathcal{E} / \hbar^{2}} r_{b}\right)-(K \leftrightarrow I)\right] \\
\times & \frac{I_{\nu}\left(\sqrt{2 m \mathcal{E} / \hbar^{2}} r_{a}\right)}{I_{\nu}\left(\sqrt{2 m \mathcal{E} / \hbar^{2}} a\right)} .
\end{aligned}
$$

The corresponding bound-state energy spectra are given by the equation

$$
I_{l+\left|k+\beta_{0}\right|+1 / 2}\left(\sqrt{2 m \mathcal{E} / \hbar^{2}} a\right)=0 .
$$

We again see that the presence of the flux line in the circular billiard simply changes the order of the Bessel functions. The energy spectra are determined by the zero points of the modified Bessel function. This quantum effect may be detected by the experiment. It has of much interest in the mesoscope systems. For the non-relativistic quantum AB billiard system, the exact Green function is obtained by replacing the pseudoenergy $\mathcal{E}$ with $-E$.

\section{Concluding remarks}

In this paper, the Green functions of the relativistic two- and three-dimensional AB systems are given by the path integral approach. The results are separated into angular and radial 
parts. From the radial parts, the Green functions of the relativistic two- and three-dimensional quantum $\mathrm{AB}$ billiard systems are obtained via the closed formula of the Dirichlet boundary condition given by the $\delta$-function perturbation. The energy spectra are determined by the zeros of the modified Bessel function involving the partial wave expanded Green function of the unperturbed $\mathrm{AB}$ systems. The $\mathrm{AB}$ system serves as the prototype of arbitrary systems bounded by the spherical Dirichlet boundary condition. There is an interesting effect in mesoscope systems related to our results for the non-relativistic case. Such effect can be described by the AB magnetic field surrounded by a spherically shaped $\delta$-function. Its Green function is given by $[4,5,7]$

$$
G^{(\delta)}\left(r_{b}, r_{a} ; E\right)=G\left(r_{b}, r_{a} ; E\right)-\frac{G\left(r_{b}, a ; E\right) G\left(a, r_{a} ; E\right)}{G(a, a ; E)-\hbar / \alpha a^{(D-1)}}
$$

with $G$ being the radial Green function without $\delta(r-a)$ potential and $\alpha$ the interacting strength of $\delta$-function. In two-dimensional case, with the result of equation (2.20), equation (4.1) yields for $r_{b}>a>r_{a}$

$$
G^{(\delta)}\left(r_{b}, r_{a} ; E\right)=-\frac{2 m}{\alpha a} \frac{I_{\left|n+\beta_{0}\right|}\left(\sqrt{-2 m E / \hbar^{2}} r_{a}\right) K_{\left|n+\beta_{0}\right|}\left(\sqrt{-2 m E / \hbar^{2}} r_{b}\right)}{(2 m / \hbar) I_{\left|n+\beta_{0}\right|}\left(\sqrt{-2 m E / \hbar^{2}} a\right) K_{\left|n+\beta_{0}\right|}\left(\sqrt{-2 m E / \hbar^{2}} a\right)-\hbar / \alpha a} .
$$

Energy spectra $E_{n}$ of bound states are determined by the equation

$$
\frac{\hbar^{2}}{2 m \alpha a}=I_{\left|n+\beta_{0}\right|}\left(\sqrt{-2 m E_{n} / \hbar^{2}} a\right) K_{\left|n+\beta_{0}\right|}\left(\sqrt{-2 m E_{n} / \hbar^{2}} a\right) .
$$

From the asymptotic behaviour of $I_{\alpha}(\alpha z) K_{\alpha}(\alpha z)$ for $\alpha \rightarrow \infty[10, \mathrm{p} 378]$

$$
I_{\alpha}(\alpha z) K_{\alpha}(\alpha z) \approx \frac{1}{2 \alpha \sqrt{1+z^{2}}}
$$

we have

$$
\frac{\hbar^{2}}{m \alpha a} \approx\left(\left|n+\beta_{0}\right|+\frac{2 m\left|E_{n}\right| a^{2}}{\hbar^{2}}\right)^{-1 / 2}<\frac{1}{\left|n+\beta_{0}\right|} .
$$

This implies that only finite bound states exist and the upper bound is given by

$$
\left|n+\beta_{0}\right|<\frac{m \alpha a}{\hbar^{2}} .
$$

We see that the AB effect not only changes the energy levels but also changes the number of bound states. On the other hand, the radius of a thin-walled cylinder also affects the number of energy levels. For the three-dimensional case, with the radial Green function (3.26), we have the Green function of semi-transparent wall for $r_{b}>a>r_{a}$

$$
\begin{aligned}
G^{(\delta)}\left(r_{b}, r_{a} ; E\right) & =-\frac{1}{\sqrt{r_{b} r_{a}}} \\
& \times \frac{\frac{2 m}{\alpha a^{2}} I_{l+\left|k+\beta_{0}\right|+1 / 2}\left(\sqrt{-2 m E / \hbar^{2}} r_{a}\right) K_{l+\left|k+\beta_{0}\right|+1 / 2}\left(\sqrt{-2 m E / \hbar^{2}} r_{b}\right)}{\frac{2 m}{\hbar a} I_{l+\left|k+\beta_{0}\right|+1 / 2}\left(\sqrt{-2 m E / \hbar^{2}} a\right) K_{l+\left|k+\beta_{0}\right|+1 / 2}\left(\sqrt{-2 m E / \hbar^{2}} a\right)-\frac{\hbar}{\alpha a^{2}}} .
\end{aligned}
$$

The energy levels of bound states are determined by

$$
\frac{\hbar^{2}}{2 m \alpha a}=I_{l+\left|k+\beta_{0}\right|+1 / 2}\left(\sqrt{-2 m E_{n} / \hbar^{2} a}\right) K_{l+\left|k+\beta_{0}\right|+1 / 2}\left(\sqrt{-2 m E_{n} / \hbar^{2}} a\right) \text {. }
$$


A similar analysis of the two-dimensional case gives the upper bound of eigenvalue for the $\left(l+\left|k+\beta_{0}\right|+1 / 2\right) \rightarrow \infty$ as

$$
\frac{\hbar^{2}}{m \alpha a} \approx\left(l+\left|k+\beta_{0}\right|+1 / 2+\frac{2 m\left|E_{n}\right| a^{2}}{\hbar^{2}}\right)^{-1 / 2}<\frac{1}{l+\left|k+\beta_{0}\right|+1 / 2}
$$

i.e.

$$
\left(l+\left|k+\beta_{0}\right|+1 / 2\right)<\frac{m \alpha a}{\hbar^{2}} .
$$

It is easy to see that the number of bound states of the three-dimensional case is greater than that of the two-dimensional one for an extra degree of freedom of quantum number. These results provide a reasonable basis for explaining the dependence of the electron wavefunctions on the vector potential of the $\mathrm{AB}$ magnetic fields when an electron penetrates the spherical cylinder.

\section{Acknowledgments}

The paper is supported by the National Science Council of Taiwan under contract number NSC89-2112-M-009-065.

\section{References}

[1] Kleinert H 1996 Phys. Lett. A 21215

[2] Lin D H 1998 J. Phys. A: Math. Gen. 314785 Lin D H 1999 J. Math. Phys. 401246

[3] Lin D H 1997 J. Phys. A: Math. Gen. 303201 Lin D H 1998 J. Phys. A: Math. Gen. 317577

[4] Grosche C 1993 Phys. Rev. Lett. 711

[5] Lin D H 1997 J. Phys. A: Math. Gen. 304365

[6] Erdélyi A, Magnus W, Oberhettinger F and Tricomi F G (ed) 1954 Table of Integral Transforms vol 1 (New York: McGraw-Hill)

[7] Grosche C and Steiner F 1998 Handbook of Feynmann path integrals Springer Tracts in Modern Physics vol 145 (Berlin: Springer)

[8] Kleinert H 1995 Path Integrals in Quantum Mechanics, Statistics and Polymer Physics (Singapore: World Scientific)

[9] Magnus W, Oberhettinger F and Soni R P 1966 Formulas and Theorems of the Special Function of Mathematical Physics (Berlin: Springer)

[10] Abramowitz M and Stegun I A (ed) 1964 Handbook of Mathematical Functions (Washington, DC: US Govt Printing Office) 